\title{
Regional differences in the health status of Chinese residents: 2003-2013
}

\author{
ZHAO Xueyan, WANG Weijun, WAN Wenyu \\ College of Geography and Environment Science, Northwest Normal University, Lanzhou 730070, China
}

\begin{abstract}
Health inequality is an increasing concern worldwide. Using the coefficient of variation, Theil index, exploratory spatial data analysis, and spatial panel econometric model, we examined the regional inequality, spatio-temporal dynamic patterns, and key factors in the health status of Chinese residents from 2003 to 2013. We found that China's residential health index $(\mathrm{RHI})$ decreased from 0.404 to 0.295 in 2003-2013 at an annual rate of $2.698 \%$. Spatially, resident health status, based on the $\mathrm{RHI}$, has improved faster in the western region than in the eastern and central regions. Inequality in resident health status continued to increase between 2003 and 2013; inequality between regions decreased, but health status inequality expanded within regions. Furthermore, disparities in health status grew faster in western regions than in the eastern and central regions. The spatial distribution of resident health status formed a "T-shaped" pattern across China, decreasing from east to center then to the west with a symmetric decrease north and south. Using the change in Moran's I from 2003 to 2008 and 2013, we found that the distribution of resident health status across China has narrowed. All the hot spots and cold spots have decreased, but they are also stable. Resident health status formed a stable cold spot in the western regions, while the east coastal area formed a stable hot spot. Selected explanatory variables have significant direct impacts on resident health status in China: increasing per capita GDP, per capita spending on health, and urbanization, and improving environmental quality all lead to better resident health status. Finally, we highlight the need for additional research on regional inequality of resident health status across multiple time, spatial, and factor domains.
\end{abstract}

Keywords: residents; health status; regional inequity; influencing factors; China

\section{Introduction}

Health, the basis of human survival and development, is related to quality of life, national security, and social stability. As the socio-economy and medical technology have developed, the national health status of different countries has all improved. However, upper-class populations have gained greater benefits from this improvement, which has resulted in health inequities between and within countries (Smyth, 2008; Yang et al., 2010; Brinda et al., 2016). Currently, health inequity has become a central issue affecting human development

Received: 2017-06-12 Accepted: 2017-08-02

Foundation: National Natural Science Foundation of China, No.41661115, No.41361106; The Open Fund of Key Laboratory of Ecohydrology of Inland River Basin, Chinese Academy of Sciences, No.KLEIRB-2S-16-03

Author: Zhao Xueyan (1971-), Professor, specialized in ecological economics. E-mail: zhaoxy@nwnu.edu.cn 
(Kirby, 2005; Braveman, 2006). The World Health Organization (WHO) has strongly urged nations to narrow the gap in health disparities between different groups of people and different regions within a country. Enjoying good health should be a main social goal of governments worldwide.

Since the reform and opening up, access to health care services in China has been significantly enhanced, and resident health status has improved significantly. Particularly in recent years, reforms in the health care system and continuous increases in health resources have rapidly changed the status of resident health, which is clear from the following data. Life expectancy has been extended from 69 years in 1990 to 75 years in 2010, mortality of children under 5 years fell from 53.9 per thousand in 1990 to 11.7 per thousand in 2014 and the maternal mortality rate dropped from 80 per 100,000 in 1990 to 21.7 per 100,000 persons in 2014 (NHFPC, 2015). However, there are still significant regional differences in health status, which pose severe challenges to building a harmonious society in China. To address this disparity, the Ministry of Health pointed out in 2008 that "the health equity of people should be set as an indicator to measure social justice and fairness" in the "Healthy China 2020" report. Moreover, in the fifth plenary session of the 18th Central Committee, the construction of "Healthy China" was proposed as a national strategy, which stressed the necessity of integrating health into all policies, changing the pattern of health development, and promoting and protecting the health of the entire nation. Therefore, understanding regional differences in the status of resident health has important significance for achieving the "Healthy China" objective.

International research on health equity began in the 1970s. In 1977, the British government set up a "Health Inequity" research group and released "The Black Report" in August 1980. The report documented social health inequities, and attributed the health disparities between different social classes to the differences in socio-economic environment. The report has attracted widespread attention about health equity. Published research has shown that health inequities are closely related to socio-economic status and that the health status of groups with higher socio-economic status is significantly superior to those with lower socio-economic status, and this trend has not changed spatially or temporally (Mackenbach et al., 2008). For example, Acheson (1998) found that life expectancy remains unequal between social classes, even in countries with generally high health status. Zatonski (2007) pointed out that the biggest challenge to the continued socio-economic development of Europe was the health gap between the east and the west. Pearce et al. (2006) claimed that health inequities between different regions in New Zealand had become more extreme in the early 21 st century and the gap was growing. Furthermore, Hong and Ahn (2011) found that socio-economic inequalities between regions in South Korea worsened the health inequities between regions. Therefore, narrowing the gap in health inequities between different regions requires a more equitable distribution of income and higher average incomes. Since the 1990s, the coupled growing gaps between social classes and improving health has highlighted the issue of health equity in China. Health inequity between diverse groups (e.g., between ethnic, social, and class groups) and between urban and rural areas has been studied; however large-scale regional differences in health inequity are less well understood. Previous studies have concentrated on measuring regional health inequity based on indicators such as the infant mortality rate (Ming, 2007), self-assessment health index (Xie 
and $\mathrm{Tu}, 2011$ ), and compound health index (Fang et al., 2010) using the coefficient of variation, extreme rate, Theil index, and concentration index. These scholars found significant differences in the status of resident health in different regions of China, with better status in economically developed areas than in underdeveloped areas (Xie, 2011; Du and Wang, 2013; Li and Xu, 2015; Zhou et al., 2017).

In general, two problems can be found in previous studies. First, many studies have been based on static and single-year cross-section data on resident health status; this lacks sufficient study on the spatio-temporal changes in health status. Second, most studies apply descriptive statistics and clustering analysis to describe regional health inequity, but lack in-depth data on regional differences, spatial-temporal changes, and factors influencing health status. A major turning point in China's medical and health work since the reform and opening up was the year 2003. In that year, the government started constructing and began to conduct activities within the "new rural cooperative medical system." Consequently, the health of many Chinese residents began to change. Taking this turning point into consideration, this study set 2003 to 2013 as the research period and selected 2003, 2008 and 2013 three time nodes. Thirty-one Chinese provincial administrative units were set as the basic space units. We used the coefficient of variation and Theil index to analyze characteristic changes in the status of resident health and general variability. We also examined the spatial-temporal patterns of resident health status using the spatial autocorrelation method, and then determined the primary influencing factors on health status with the Spatial Econometric Model. The breadth and depth of analysis should provide an important reference to evaluate the successful implementation of the "Healthy China" strategy.

\section{Data sources and methods}

\subsection{Data sources}

The space units were the 31 provinces in China, including municipalities and autonomous regions. Perinatal mortality, maternal mortality, and incidence of infectious diseases, which reflect the health status of residents, were obtained from the 2004-2014 China Health Statistics Yearbook. Accessibility to health care resources, such as the number of beds in medical institutions per 1000 persons, the number of technical health workers per 1000 persons, and the number of hospitals per 10,000 persons and GDPPC (Gross Domestic Product Per Capita) of the provincial economic development level and urbanization rate (the proportion of urban population to total population) were acquired from the 2004-2014 China Statistical Yearbook. Provincial environmental quality metrics, such as quantity of discharged industrial wastewater and domestic sewage and industrial emissions, were obtained from the 2004-2014 China Environment Statistical Yearbook.

\subsection{Methods}

2.2.1 Measuring the health status of residents

The United Nations Millennium Development Goals proposed "to reduce child mortality, improve maternal health, and fight against HIV and other diseases." Some data was limited, for example, the absence of provincial data on child mortality under age 5 , which is a com- 
monly used indicator. Therefore, we only selected perinatal mortality, maternal mortality, and incidence of infectious diseases to reflect resident health status. Among them, the incidence of infectious diseases was represented by the incidence rate of A or B class notifiable infectious diseases. Perinatal mortality, maternal mortality, and incidence of infectious diseases are negative indicators; greater values indicate worse health status. To comprehensively evaluate the resident health status in different regions, these described indicators were first normalized using the extreme value normalization method. Then, the weight of the index was determined using the entropy method (Chen et al., 2009). Finally, the weighted average method was applied to calculate the residents' health index in different provinces.

$$
H_{i j}=\sum_{j=1}^{3} Z_{i j} \cdot w_{i j}
$$

where $H_{i j}$ is the residents' health index (RHI) in $i$ province, $Z_{i j}$ is the normalized value of the $j$ th indicator in $i$ province, and $w_{i j}$ is the weight of the $j$ th indicator. The weights of perinatal mortality, maternal mortality, and incidence of infectious diseases are $0.34,0.31$, and 0.35 , respectively. Therefore, smaller values of $H_{i j}$ indicate better health status.

\subsubsection{Measuring regional differences in resident health status}

The coefficient of variation and Theil index were used to measure the regional differences in resident health status.

$$
C_{v}=\frac{1}{\bar{h}} \sqrt{\sum_{i=1}^{n} \frac{\left(h_{i}-\bar{h}\right)}{(n-1)}}
$$

where $C_{v}$ is the coefficient of variation, $n$ is the number of provinces, $h_{i}$ is the residents' health index in $i$ province and $\bar{h}$ is the average value of all $h_{i}$. Larger coefficients of variation indicate greater differences.

The Theil index can resolve the overall differences in the health status into the differences within regions and the ones between regions.

$$
\begin{gathered}
\text { Theil }=\sum_{i=1}^{n} T_{i} \ln \left(n T_{i}\right)=T_{W R}+T_{B R} \\
T_{W R}=\sum_{i=1}^{n_{d}} T_{i} \ln \left(n_{d} \frac{T_{i}}{T_{d}}\right)+\sum_{i=1}^{n_{z}} T_{i} \ln \left(n_{z} \frac{T_{i}}{T_{z}}\right)+\sum_{i=1}^{n_{x}} T_{i} \ln \left(n_{x} \frac{T_{i}}{T_{x}}\right) \\
T_{B R}=T_{d} \ln \left(T_{d} \frac{n}{n_{d}}\right)+T_{z} \ln \left(T_{z} \frac{n}{n_{z}}\right)+T_{x} \ln \left(T_{x} \frac{n}{n_{x}}\right)
\end{gathered}
$$

where $T_{W R}$ is the differences within regions, $T_{B R}$ is the differences between regions, and $n$ is the number of provinces; $n_{d}, n_{z}$, and $n_{x}$ are the number of eastern, central, and western provinces, respectively; $T_{i}$ is the ratio of residents' health index to the national average index; $T_{d}, T_{z}$, and $T_{x}$ are the ratios of residents' health index in the eastern, central, and western regions to the national average index.

\subsubsection{Measuring spatial patterns of resident health status}

Exploratory Spatial Data Analysis (ESDA) was applied to analyze the distribution patterns of the resident health status in China. The global spatial Moran's $I$ was used to determine whether the resident health status distributions were clustered, dispersed, or random (Wang 
and $\mathrm{Xu}, 2017)$. The formula is as follows:

$$
\text { Moran's } I=\frac{\sum_{i=1}^{n} \sum_{j=1}^{n} W_{i j}\left(Y_{i}-\bar{Y}\right)\left(Y_{j}-\bar{Y}\right)}{S^{2} \sum_{i=1}^{n} \sum_{j=1}^{n} W_{i j}}
$$

where $n$ is the total number of observations, $Y_{i}$ is the observed value of resident health status at $i$ position, $W_{i j}$ is the spatial weight matrix (spatial adjacent: 1 , non-adjacent: 0 ), $S^{2}$ is the variance of the attribute value, and $\bar{Y}$ is the average value of $Y_{i}$. At a given level of significance, positive Moran's $I$ values indicate clustering of regions with similar health status. Conversely, negative Moran's $I$ indicate that adjacent areas have significant differences in resident health status, i.e., good and poor health statuses are significantly spatially dispersed. Normally, Moran's $I$ is examined with the Z-test. Positive and significant $Z$ values indicate a positive spatial autocorrelation. Negative and significant $Z$ values indicate that there is negative spatial autocorrelation. $Z$ value of zero indicates an independent and random distribution.

$$
Z(I)=\frac{I-E(I)}{\sqrt{\operatorname{Var}(I)}}
$$

where $E(I)$ is mathematical expectation and $\operatorname{Var}(I)$ is the variance.

Getis-Ord $\mathrm{G}^{*}$ was adopted to reflect the spatial dependency and heterogeneity of the resident health status. The formula can be expressed as:

$$
G_{i}^{*}(d)=\sum_{i=1}^{n} W_{i j}(d) Y_{i} / \sum_{i=1}^{n} Y_{i}
$$

where a significantly positive $G^{*}{ }_{i}(d)$ value indicates that the observed value is relatively high around area $i$, which indicates a hot-spot area; other values of $G^{*}{ }_{i}(d)$ indicate cold-spots. $Y_{i}$ is the observed value of resident health status in region $i$ and $W_{i j}$ is the spatial weight matrix, where the space adjacent value is 1 and the non-adjacent value is 0 .

\subsubsection{Spatial regression analysis to evaluate the influencing factors on resident health status}

Because the traditional econometric model does not nest the spatial interaction effect, it may lead to errors in the model setting and estimation results. Instead, a spatial panel model with both nested spatial and time effects makes the spatial regression model set more practical. The spatial panel model also better clarifies the influence of the selected factors on the spatial-temporal patterns of resident health status. At present, commonly used spatial regression models include the Spatial Lag Model (SLM), Spatial Error Model (SEM), and Spatial Durbin Model (SDM) (Lesage and Pace, 2009). Among them, the SDM has nested spatial dependencies, and the independent variables and error terms are unaffected by the spatial dependence of missing variables (Lee and $\mathrm{Yu}, 2010$ ). Therefore, the SDM was used to analyze the effect of key human factors on spatial-temporal changes in resident health status.

The provincial unit was set as $i=1, \ldots, 31$, and the time series was set as $t=1, \ldots, 11$ (from 2003 to 2013). The SDM model, which analyses the relationship between key human factors and resident health status can be expressed as: 


$$
y_{i t}=\delta \sum_{j=1}^{n} w_{i t} y_{j t}+\beta \sum_{j=1}^{n} r w_{i j} x_{i, j, t-1}+\mu_{i}+\lambda_{t}+\varepsilon_{i t}
$$

where $\delta$ represents the spatial regression coefficient, $w_{i j}$ represents an element in the spatial weight matrix $W, y_{j t}$ represents the observed value of the resident health status in $j$ unit at time $t$ and $x_{(i, j, t-1)}$ is the row vector $(1, k)$ of independent variable in $i$ unit at time $t-1 . \lambda$ is the column vector of $k$ dimension, which represents the coefficient of the spatial hysteresis interpretation variable. $\mu_{i}$ represents the spatial fixed effect, which controls all variables with space-fixed and time-independent. $\lambda_{i}$ represents the time fixed effect, which controls all variables with time-fixed and space-independent. $\varepsilon_{i t}$ represents the spatial autocorrelation error term.

\section{Regional differences in Chinese resident health status and changes in spatio-temporal patterns}

\subsection{Changes in regional differences in resident health status}

From 2003-2013, the residents' health index (RHI) of Chinese residents decreased from 0.404 to 0.295 , indicating that the health status improved $26.98 \%$ (Figure 1). However, due to the spread of the SARS epidemic in 2003 and an imperfect public health service system, from 2003 to 2005, the Chinese RHI increased from 0.404 to 0.464 , indicating a health status deteriorated $14.91 \%$. Since then the public health system has improved with reforms in the medical and health system and increasing investments in medical and health. This resulted in the Chinese RHI dropping to 0.295 from 0.464 with a corresponding health status improvement of $36.51 \%$. From 2003 to 2013, the full implementation of the new rural cooperative medical system, the central government's subsidies, and increasing investments in the western region resulted in significant advances in the health status of western Chinese residents. The RHI in the western region decreased from 0.558 to 0.353 and health status improved $32.26 \%$; this is in contrast to the central and eastern regions, where health status improved $17.42 \%$ and $23.74 \%$, respectively. However, despite these gains, health status in China continued to show an "East-Centre-West" stepwise declining pattern.

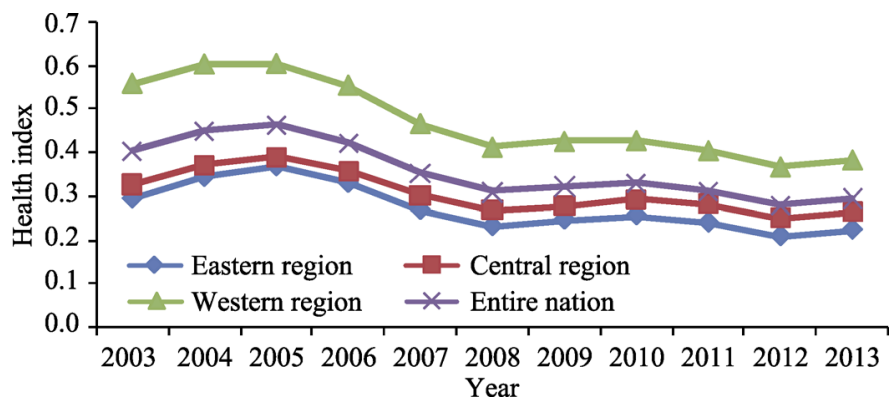

Figure 1 Trends in the residents' health index (RHI) in China from 2003 to 2013

From 2003 to 2013, the differences in resident health status between provinces were large and the overall trend widened; the coefficient of variation increased from 0.369 to 0.495 , a growth of $27.06 \%$, and the Theil index increased from 0.0619 to 0.0832 , an increase of 
$34.47 \%$ (Figure 2). The SARS epidemic in 2003 significantly impacted resident health status and differences in resident health status between provinces from 2003 to 2005 began to narrow. Subsequently, provincial differences widened and fluctuated. The Theil index showed that the evolution of differences within regions (intra-zones) coincided with overall differences. More specifically, from 2003 to 2007, differences between regions (inter-zones) were wider than those within regions and overall differences were primarily caused by differences between regions, with an average contribution rate of $61.45 \%$. Then from 2008 to 2013 , the differences within regions were greater than those between regions, and the overall differences were primarily caused by differences within regions, with an average contribution rate of $60.15 \%$. In general, the differences in the resident health status between regions tended to shrink, while differences within regions tended to expand. However, there were significant differences in trends of the variation in resident health status in the eastern, central, and western regions. That is, while the differences within the eastern and central regions were growing, the fluctuation was relatively stable and the amplitude was small. In contrast, the differences within western region rapidly expanded, the coefficient of variation increased $114.21 \%$ and the Theil index increased $274.18 \%$; the differences in health status of residents of the western provinces became increasingly significant.

(a) Variable coefficient

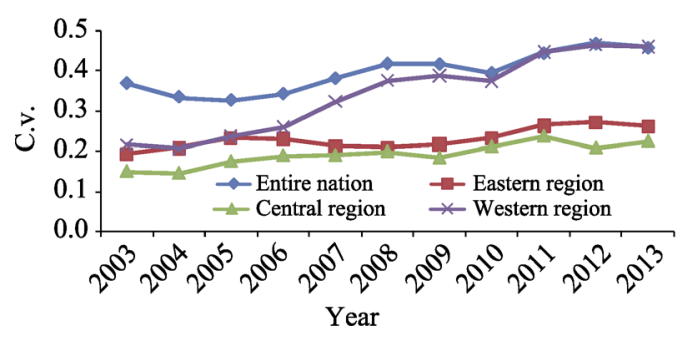

(b) Theil index

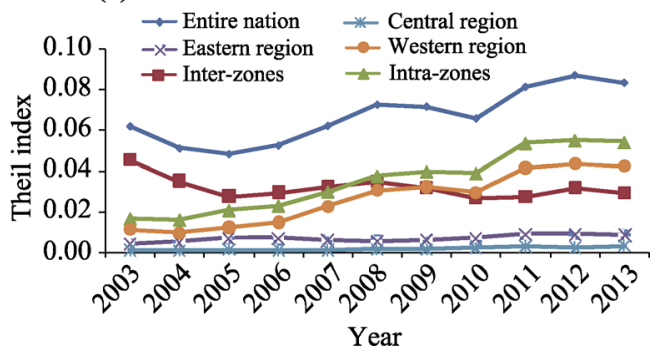

Figure 2 Regional differences in resident health status in China from 2003-2013 based on (a) the variable coefficient and (b) the Theil index

\subsection{Changes in spatial distribution of resident health status}

To present the spatial distribution of Chinese resident health status visually for 2003, 2008, and 2013, the 31 provinces were divided into five groups based on their RHI and plotted with natural breakpoint classification method using ArcGIS software. These groups were high, above-medium, medium, below-medium, and low health status regions.

Between 2003 and 2013, the health status of Chinese residents changed significantly at the provincial scale (Figures 3 and 4). From 2003 to 2008, 22.58\% of provinces transferred to a high status and $19.35 \%$ to a low status. The status transformations for provinces were complex and did not improve systematically. That is, $9.68 \%$ of provinces directly transformed to a high health status, $12.90 \%$ of provinces gradually improved in status, and $6.45 \%$ of provinces directly transformed to a low health status, $12.90 \%$ of provinces gradually declined in status. From 2008 to 2013, status transformations were simpler, and all transformations were gradual, successive migrations to higher or lower status. Of these, $19.35 \%$ of provinces transferred to a high status and only $6.45 \%$ of provinces were to a low status. Compared with 2003-2008, the number of high, below-medium, and low health status 
provinces decreased $33.33 \%, 75.0 \%$, and $60.0 \%$, respectively, while above-medium and medium health status provinces increased $11.11 \%$ and $100 \%$, respectively. Generally, the high and above-medium health status provinces were primarily in the eastern region for 2003-2013, although the proportion of eastern provinces decreased slightly. The central region was dominated by above-medium and medium health status provinces. The western region was dominated by provinces that changed from low and below-medium to medium and above-medium health status. Despite improvements in the overall health status, the general feature of the "East-Centre-West" stepwise declining pattern remained unchanged and the differences within the eastern and western regions remained (Figure 4).

In addition to the general spatial trends, the health status distributions also changed. From 2003 to 2008 (Figure 3), the high health status regions shrank sharply, and changed from relatively dispersed to concentrated, forming the contiguous high health status area of Jiangsu-Shandong-Shanghai. The spatial range of above-medium health status regions slightly expanded and formed three contiguous areas, Beijing-Tianjin-Hebei-Liaoning, Sichuan, and Hunan-Hubei-Jiangxi-Anhui-Zhejiang. The spatial scope of the medium health status regions expanded largely, shifting from relative dispersed to more concentrated areas; there was a contiguous area of Fujian-Guangdong-Guangxi-Yunnan-Hainan and a wedge-shaped area of Inner Mongolia-Heilongjiang-Jilin-Shanxi-Shaanxi-Cho ngqing. Both the below-medium and low health status regions declined largely, with

(a) 2003

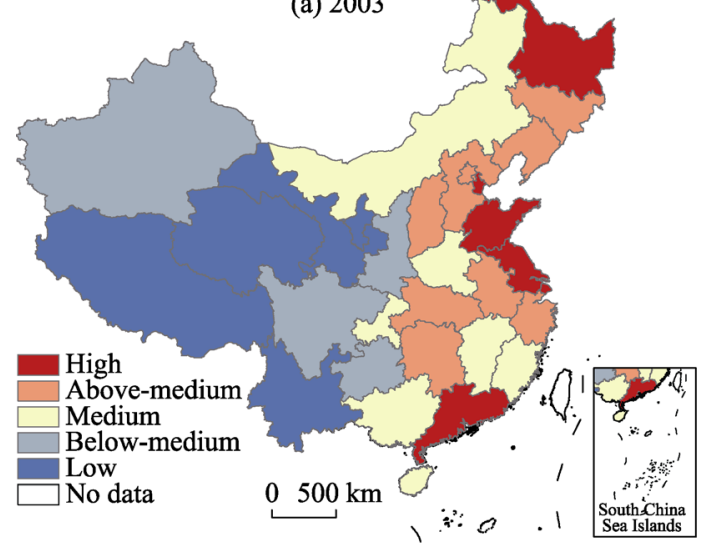

(b) 2008

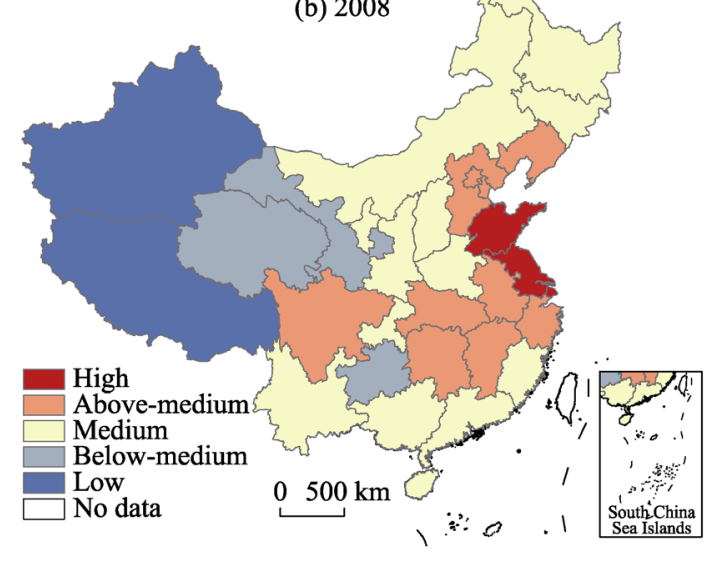

(c) 2013

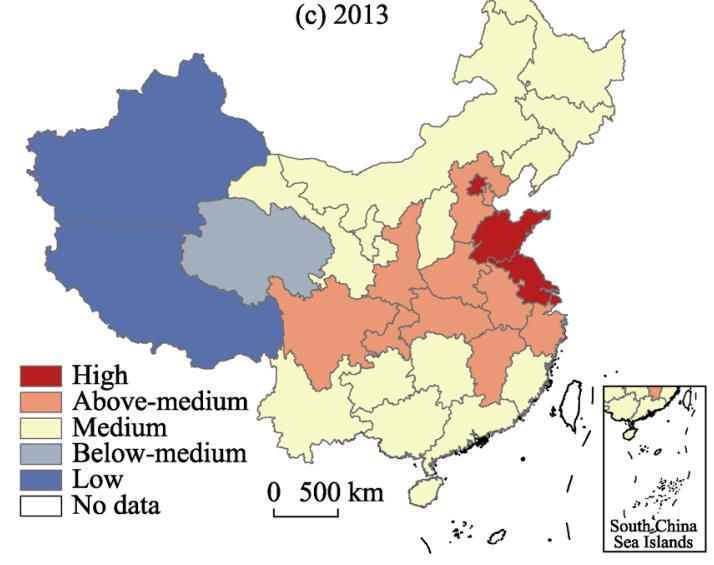

Figure 3 The spatial distribution of resident health status in China for 2003 (a), 2008 (b), and 2013 (c) the low health status regions transformed from wedge-shaped to half-surrounded below-medium health status regions. From 2008 to 2013 the spatial distributions became more concentrated. The spatial scope of high health status regions slightly expanded to include 
Beijing outside the contiguous distribution area (Jiangsu-Shandong-Shanghai) and connected with the above-medium health status regions across central China. Together, the two regions formed a "T-shaped" pattern stretching from the coast to the inland, and divided the medium-level regions into southern and northern stripes extending from east to west. The below-medium health status regions shrank slightly, but remained half-surrounded by the low health status regions; these two regions were closely grouped and located in the northwest. As a result, the spatial variation in Chinese resident health status showed a clear "East-Centre-West" stepwise declining pattern and a symmetrical declining trend toward north and south from central China.

(a) Eastern region

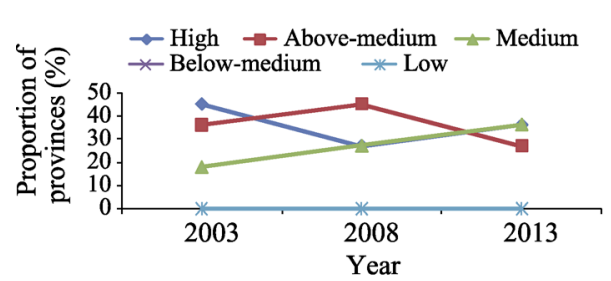

(c) Western region

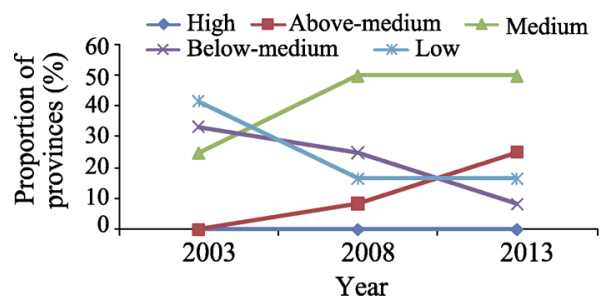

(b) Central region

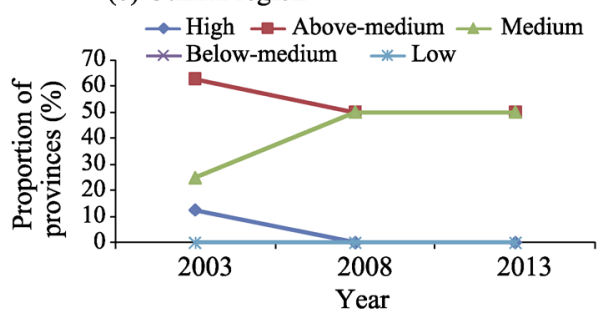

(d) Entire nation

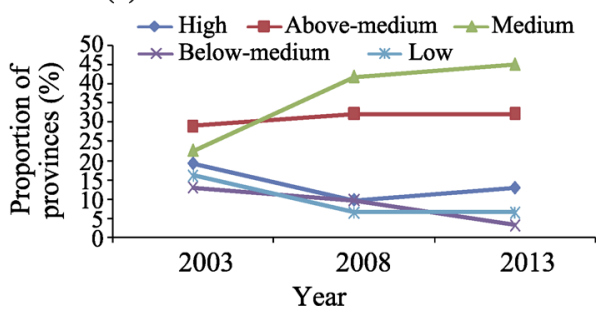

Figure 4 Resident health status in different parts of China, eastern region (a), central region (b), western region (c), and the entire nation (d), from 2003 to 2013

\subsection{Spatio-temporal changes in resident health status}

Using the RHI from each province in the ESDA method, Moran's I values were $0.377,0.268$, and 0.262 , respectively, in 2003, 2008, and 2013. The confidence level for the normal statistic Z value of Moran's $I$ was higher than 0.01 , which showed that the RHIs from 2003 to 2013 were positively spatially autocorrelated. That is, the provinces with higher resident health status tended to gather, and so did the provinces with lower resident health status. However, during this period, Moran's I value of the RHI showed a decreasing trend, indicating that the spatial autocorrelation of the resident health status weakened. From the changes in Moran's $I$ value, the spatial relationship of resident health status significantly varied from 2003 to 2008, with the change in amplitude of Moran's $I$ value of 0.109 . In comparison, the amplitude was only 0.006 from 2008 to 2013, indicating few changes and general stability in the spatial relationship of health status.

Global Moran's I values only showed that the resident health status had a significant spatial correlation for all of China, but failed to reflect local spatial information. Therefore, the Getis-Ord G* values were used to define "cold spot," "sub-cold spot," "sub-hot spot," and "hot spot" regions that reflected local spatial relationships (Figure 5). Form 2003 to 2008, 
the spatial relationship of Chinese resident health status changed largely, cold-spot regions and hot-spot regions shrank while sub-cold regions and sub-hot regions expanded. The proportion of hot-spot and cold-spot regions to all provinces dropped from $45.16 \%$ to $35.48 \%$ and from $16.13 \%$ to $9.68 \%$, respectively. Stable provinces accounted for $77.42 \%$ of the total; among these, Xinjiang-Qinghai-Tibet was a stable cold-spot region while Beijing-Tianjin-Hebei-Shandong-Henan-Hubei-Jiangxi -Jiangsu-Anhui-Shanghai-Zhejiang was a stable hot-spot region. From 2008 to 2013, the spatial relationship of Chinese resident health status changed little, the cold-spot regions remained unchanged, hot-spot regions decreased in area, and sub-cold and sub-hot regions continued to expand. The proportion of hot-spot region to all provinces reduced from $35.48 \%$ to $16.13 \%$, and stable provinces accounted for $74.19 \%$; among these, XinjiangQinghai-Tibet remained a stable cold-spot region and Shandong-Henan-Hubei-Anhui-Jiangsu-Shanghai remained a stable hot-spot region. In general, both hot-spot and cold-spot regions declined from 2003 to 2013, which indicates that the concentration trend for both high and low health status provinces weakened. This trend was particularly clear for provinces with high health status. In both periods, the percentage of stable provinces was higher than $70 \%$, indicating that the distribution of Chinese resident health status had a spatial locking or path dependence. The western region formed a large-scale cold-spot region with stability while the eastern coast was dominated by a hot-spot region with significant stability. Consequently, this analysis reinforced the overall characteristic of "East-Centre-West" stepwise decline in resident health status.
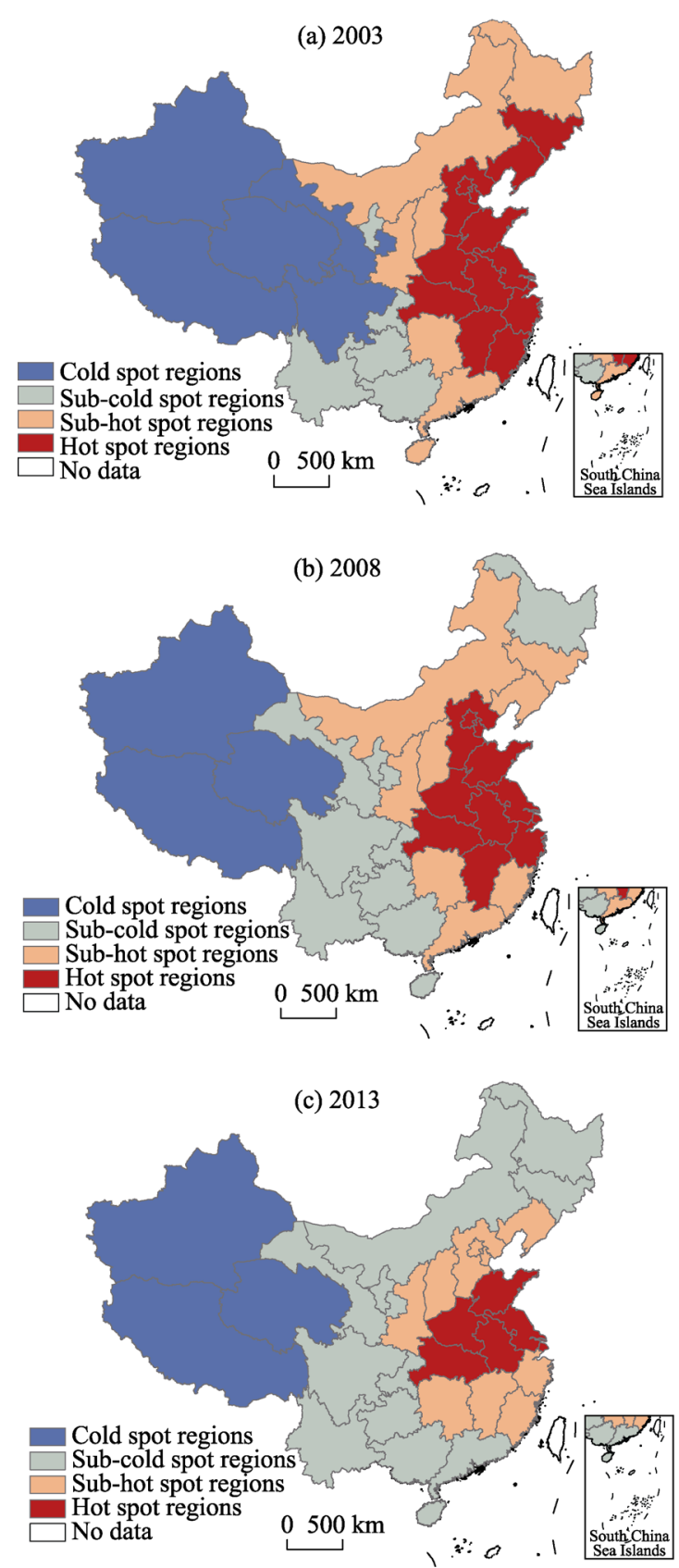

Figure 5 The spatio-temporal dynamics of the resident health status in China based on local spatial relationships in 2003 (a), 2008 (b), and 2013 (c) 


\section{Influencing factors on Chinese resident health status}

\subsection{Regional differences in key human factors}

The Commission on Social Determinants of Health within the WHO suggested that health inequities are not simply "natural" phenomena and significantly affected by unfair human factors (WHO CSDH, 2008). Some research has also shown that regional health inequities are not only the results of differences in natural factor, but are influenced by economic development (Sundmacher et al., 2011), public health expenditures ( $\mathrm{Li}$ and $\mathrm{Yu}, 2013$ ), access to health care resources (Garcia-Subirats et al., 2014; Boccolini and de Souza Jr, 2016), environmental quality (Qi, 2008; Baguma, 2017), urbanization (Van de Poel et al., 2012; Cheng and Yang, 2015; Miao and Wu, 2016), and other human factors. Therefore, we analyzed the impact of these key human factors on resident health with Spatial Durbin Model (SDM).

(1) Economic development

The level of economic development will affect the accessibility of health products and services. Poorly distributed economic development will lead to discrepancies in the quality of health products and services in different regions; thus, the effectiveness of preventive health services and disease control will also be affected, resulting in differences in resident health status (Sundmacher et al., 2011). From 2003 to 2013, China's GDPPC grew at an average annual rate of $29.75 \%$, and the average annual growth rate of the western region was as high as $45.52 \%$; China's economic development increased significantly, especially in the western region (Figure 6). During this period, the GDPPC in China also showed an "East-Centre-West" stepwise declining pattern; the coefficient of variation reduced $42.47 \%$, but with differences between the three regions. The variation coefficient of the GDPPC in the eastern and central regions tended to decline, $43.09 \%$ and $29.94 \%$, respectively, while that of the GDPPC in the western region increased $41.66 \%$. These data showed that provincial differences in economic development level as a whole had decreased, but the differences had expanded between the western provinces.

(2) Public health expenditure

Public health expenditure exerts a significant positive effect on health output, which impacts human health primarily by influencing access to health services, health insurance coverage, and public health ( $\mathrm{Li}$ and $\mathrm{Yu}, 2013$ ). From 2003 to 2013, per capita public health expenditure in China increased $78.53 \%$, and the average annual growth rate in the central region was as high as $120.39 \%$ (Figure 5). During this period, the coefficient of variation for per capita public health expenditure in China reduced $62.43 \%$. Among regions, the variation coefficient for per capita public health expenditure in the central region decreased $73.63 \%$, which was much higher than that of the eastern and western regions; however, the values of the coefficients of variation were lower than that of the eastern region. In general, provincial differences in public health expenditure in China narrowed, although the eastern provinces had more significant variations than the central and western provinces.

(3) Access to health care resources

The fair allocation of health care resources is an important factor that influences resident health equity (Zheng et al., 2015; Han et al., 2015; Mullachery et al., 2016). In this study, the number of technical health workers per 1000 persons, the number of beds in medical institutions per 1000 persons, and the number of hospitals per 10,000 persons were applied 

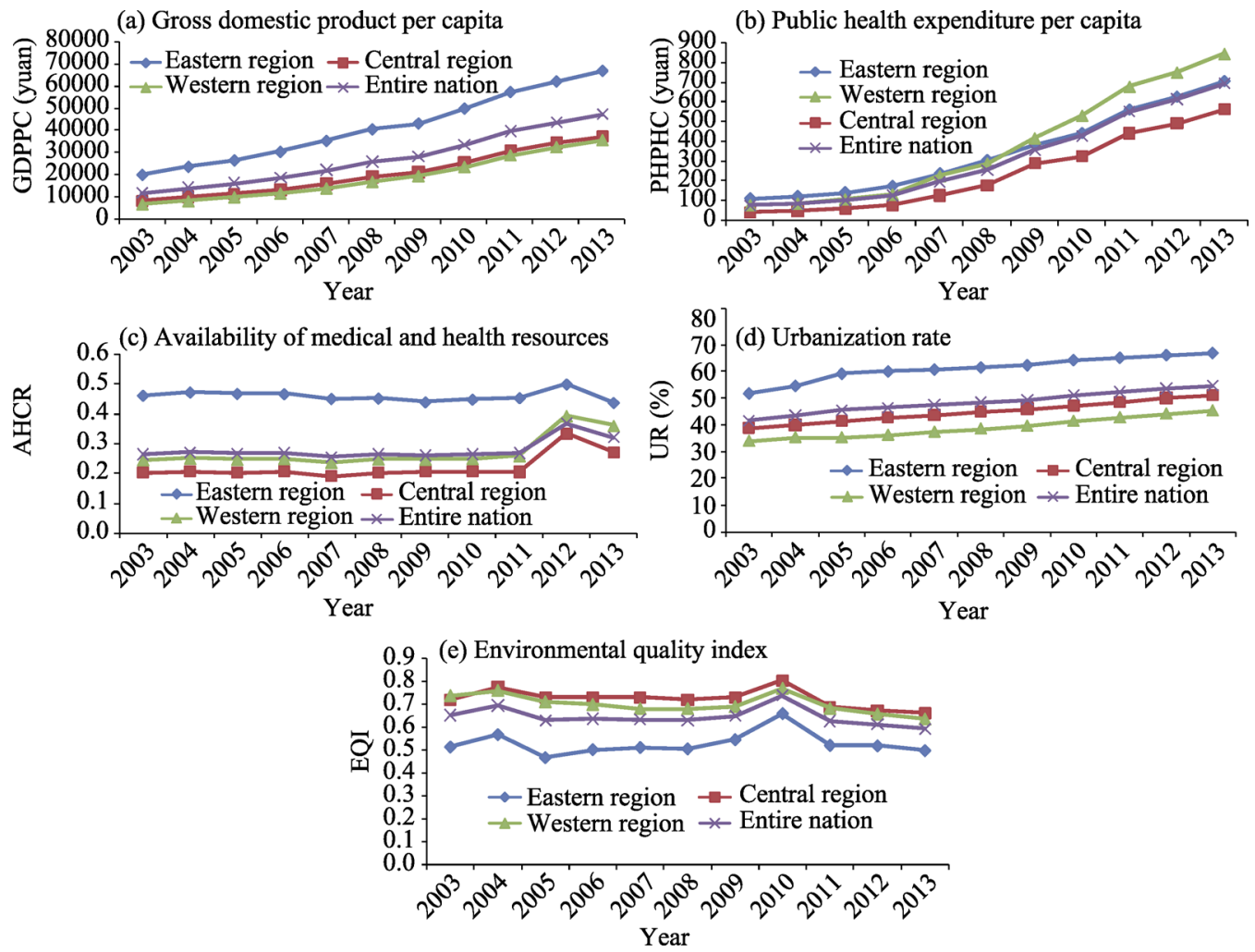

Figure 6 Trends in key human factors from 2003 to 2013, GDPPC is gross domestic product per capita (a), PCPHE is per capita public health expenditure (b), AHCR is accessibility of health care resources (c), UR is urbanization rate (d), and EQI is environmental quality index (e)

to illustrate the accessibility of health care resources ${ }^{1}$. From 2003 to 2013, the average annual growth rate (AAGR) of accessibility to health care resources in China was $2.11 \%$; in the western region, AAGR was as high as $4.76 \%$. This trend indicates that China's health care resources improved significantly, especially in the western region (Figure 5). During the period, the coefficient of variation for accessibility of health care resources in China reduced by $37.83 \%$ and it reduced by $28.59 \%$ in the central region, which was higher than in the eastern and western regions. Therefore, provincial differences in the accessibility of health care resources tended to decrease, especially in the central provinces.

(4) Urbanization

Urbanization has brought fundamental changes in social organization, family relations, and lifestyles, which exerts a significant impact on human health (Bai et al., 2012). Here, we used urbanization rate to evaluate this factor's influence on health status. The AAGR of urbanization rate in China from 2003 to 2013 was $1.32 \%$, of which the AAGR in the eastern region reached to $1.50 \%$. The urbanization level in China developed rapidly over this period, especially in the eastern region. Furthermore, although the coefficient of variation decreased $28.09 \%$ nationally, the urbanization rate had an "East-Centre-West" stepwise declining

\footnotetext{
${ }^{1}$ First, the index was standardized using the formula: $z=\left(x-x_{\min }\right) /\left(x_{\max }-x_{\min }\right)$. Second, the accessibility of health care resources was calculated using the equal weighted sum method; larger values indicate higher accessibility of health care resources.
} 
pattern and there were regional differences in the coefficient of variations. The variance coefficients for urbanization rate in the eastern and central regions declined $43.52 \%$ and $62.69 \%$, respectively, while that in the western region increased $14.02 \%$. Generally, provincial differences in the urbanization as a whole tended to decrease, except in the western region where differences in urbanization between provinces expanded.

(5) Environmental quality

The negative impact of environmental quality on health is significant (Narayan and Narayan, 2008; Li and Han, 2015), and there is a direct link between pollutants and specific diseases that can cause immediate and long-term damage to individual health and increase morbidity and mortality at a macro level ( $\mathrm{Xu}$ and $\mathrm{Chen}, 2010)$. Water and air pollution have become the primary environmental problems in China. Therefore, in this study, wastewater discharge per capita (industrial wastewater + domestic sewage) and waste gas per capita were used to represent the environmental quality ${ }^{2}$. From 2003 to 2013, the environmental quality index in China fell $1.17 \%$ annually, and the average annual decline in the western region was as high as $1.36 \%$; China's environmental quality generally worsened, especially in the western region. During this period, the environmental quality index in China maintained a "Centre-West-East" decreasing trend. The coefficient of variation decreased $6.25 \%$ nationally, but was different in each region; the variation coefficient of the environmental quality index for the eastern and central regions declined $51.12 \%$ and $12.50 \%$, respectively, while it increased $73.81 \%$ in the western region. Generally, provincial differences in environmental quality tended to decrease, although differences in the western provinces expanded.

\subsection{The impact of human factors on resident health status}

Pearson correlation analysis was used to test the relationship between these human factors and the resident's health index (Table 1). The results showed that the GDPPC and urbanization rate maintained a strongly negative correlation with the RHI, which indicated that higher provincial GDPPC and urbanization likely resulted in better resident health status. That is, economic and urban development effectively changed health status. Per capita public health expenditure and the RHI also had a significantly negative correlation for five years; regions with more public health expenditure provided better health care services, which in turn, actively improved resident health. The environmental quality index had a significant negative correlation with the residents' health index for six years, indicating that better

Table 1 Pearson correlation coefficients between RHIs and key human factors

\begin{tabular}{lccccccccccc}
\hline Variable & 2003 & 2004 & 2005 & 2006 & 2007 & 2008 & 2009 & 2010 & 2011 & 2012 & 2013 \\
\hline GDPPC & $-0.508^{* *}$ & $-0.486^{* *}$ & $-0.422^{* *}$ & $-0.453^{* *}$ & $-0.441^{* *}$ & $-0.437^{* *}$ & $-0.405^{* *}$ & $-0.425^{* *}$ & $-0.448^{* *}$ & $-0.418^{* *}$ & $-0.413^{* *}$ \\
PCPHE & -0.000 & -0.078 & -0.190 & -0.140 & -0.309 & -0.218 & $-0.427^{* *}$ & $-0.498^{* *}$ & $-0.452^{* *}$ & $-0.457^{* *}$ & $-0.454^{* *}$ \\
AHCR & -0.261 & -0.184 & -0.104 & -0.125 & -0.15 & -0.132 & -0.12 & -0.133 & -0.112 & -0.168 & -0.165 \\
UR & $-0.489^{* *}$ & $-0.437^{* *}$ & $-0.493^{* *}$ & $-0.537^{* *}$ & $-0.535^{* *}$ & $-0.541^{* *}$ & $-0.522^{* *}$ & $-0.512^{* *}$ & $-0.524^{* *}$ & $-0.550^{* * *}$ & $-0.546^{* * *}$ \\
EQI & $-0.500^{* *}$ & $-0.416^{* *}$ & -0.235 & $-0.404^{* *}$ & $-0.477^{* *}$ & $-0.370^{* *}$ & -0.372 & -0.178 & $-0.327^{*}$ & -0.251 & -0.260 \\
\hline
\end{tabular}

Note: ${ }^{* * *} p<0.001 ;{ }^{* *} p<0.05 ;{ }^{*} p<0.1$

\footnotetext{
${ }^{2}$ First, the index was standardized using the formula: $z=\left(x_{\max }-x\right) /\left(x_{\max }-x_{\min }\right)$. Second, the index for environmental quality was calculated using the equal weighted sum method; larger values indicate better environmental quality.
} 
environmental quality resulted in maintenance of good resident health status. Although the accessibility of the health care resources negatively correlated with the RHI, it was insignificant.

There was a significant spatial autocorrelation in the health status of Chinese residents, which indicated a spatial interaction effect in health status across different provinces. Therefore, SDM with random effect was selected to explain the influences of each human factor on resident health status. The estimated SDM results are shown in Table 2. From Table 2, the GDPPC, per capita public health expenditure (PCPHE), urbanization rate (UR), and environmental quality index (EQI) passed the significance test at 5\%, while the accessibility of health care resources (AHCR) failed. Therefore, economic development, public health expenditure, urbanization, and environmental quality were key factors affecting the spatio-temporal distribution of resident health status. More specifically, the elastic coefficient of GDPPC, PCPHE, UR, and EQI were $-0.017,-0.013,-0.129$, and -0.012 , respectively, which indicated that these four factors had a positive effect on the resident health status. Therefore, economic development, increased public health expenditure, higher urbanization, and better environmental quality could effectively improve resident health status. In addition, the elastic coefficients of spatial lags of GDPPC and environmental quality were -0.037 and -0.081 , respectively, but that of urbanization rate was 0.093 . Therefore, improving the economic development level and the environmental quality in neighboring provinces would have a positive impact on the resident health status in a province, while increasing urbanization in adjacent provinces would exert a negative impact on resident health in a province.

Table 2 Key human factor parameters estimated using SDM

\begin{tabular}{|c|c|c|c|c|c|c|c|}
\hline Variable & $\begin{array}{c}\text { Elastic } \\
\text { coefficient }\end{array}$ & $T$ value & $P$ value & Variable & $\begin{array}{c}\text { Elastic } \\
\text { coefficient }\end{array}$ & $T$ value & $P$ value \\
\hline $\operatorname{lnGDPPC}$ & $-0.017 * *$ & -2.39 & 0.017 & $\mathrm{~W}^{*} \ln \mathrm{GDPPC}$ & $-0.037 * * *$ & -2.79 & 0.005 \\
\hline $\ln \mathrm{PCPHE}$ & $-0.013 * *$ & -1.98 & 0.049 & $\mathrm{~W}^{*} \ln \mathrm{PCPHE}$ & 0.003 & 0.48 & 0.630 \\
\hline $\ln \mathrm{AHCR}$ & -0.054 & -1.36 & 0.174 & $\mathrm{~W}^{*} \ln \mathrm{AHCR}$ & $0.029 * * *$ & 2.83 & 0.005 \\
\hline $\ln U R$ & $-0.129 * *$ & -2.32 & 0.021 & $\mathrm{~W}^{*} \ln U \mathrm{R}$ & $0.093 * * *$ & 3.40 & 0.001 \\
\hline \multirow{2}{*}{$\operatorname{lnEQI}$} & \multirow{2}{*}{$-0.012 * *$} & \multirow{2}{*}{-2.08} & \multirow{2}{*}{0.035} & $\mathrm{~W}^{*} \ln \mathrm{EQI}$ & $-0.081 * * *$ & 3.99 & 0.000 \\
\hline & & & & $\mathrm{W}^{*} \ln \mathrm{RHI}$ & $0.030 * * *$ & 3.51 & 0.001 \\
\hline
\end{tabular}

Note: ${ }^{* * *} p<0.01 ;{ }^{* *} p<0.05 ;{ }^{*} p<0.1 ; \mathrm{W}^{*} \ln \mathrm{GDPPC}, \mathrm{W}^{*} \ln \mathrm{PCPHE}, \mathrm{W}^{*} \ln \mathrm{AHCR}, \mathrm{W}^{*} \ln \mathrm{lR}$ and $\mathrm{W}^{*} \operatorname{lnEQI}$ stand for the spatial lagging of GDPPC, PCPHE, AHCR, UR, and EQI respectively.

Table 3 shows that the direct effects of GDPPC, PCPHE, UR, and EQI were -0.018 , $-0.016,-0.132$, and -0.008 respectively. The direct effects of urbanization rate and GDPPC were larger and the two most important factors affecting resident health status. Every $1 \%$ increase in GDPPC, PCPHE, UR, and EQI would lead to improve resident health status $0.018 \%, 0.016 \%, 0.132 \%$, and $0.008 \%$, respectively. Due to feedback effects between provinces, there was a slight difference between the elastic coefficients of GDPPC, PCPHE, UR, and EQI and the values of their direct effects. Part of this feedback effect was derived from the spatial lagging of the RHI while the other was from the spatial lagging of the explanatory variables. For example, the elasticity coefficient of GDPPC was -0.017 and its direct effect was -0.018 . The reason for this difference was partly due to the negative statis- 
tics of the $\mathrm{W}^{*} \ln$ GDPPC coefficient and partly due to the significantly positive spatial lagging of the residents' health index (W*lnRHI). In addition, the feedback effects of GDPPC and UR were 0.001 and 0.003 , only accounting for $5.56 \%$ and $2.27 \%$ of their direct effects, clearly showing the differences in feedback effects from different human factors.

The indirect effects of GDPPC and EQI were -0.164 and -0.362 , respectively, while the indirect effects of the AHCR and UR were 0.125 and 0.410 , respectively (Table 3 ). These results indicate that GDPPC and environmental quality had negative spillover effects, while AHCR and UR had positive spatial spillover effects. Therefore, increasing economic development and improving environmental quality in the province would have a positive impact on the resident health status in neighboring provinces. In contrast, improving AHCR and UR would have a negative impact on the resident health status in neighboring provinces. For every $1 \%$ increase in GDPPC and EQI in a province would respectively increase the resident health status $0.164 \%$ and $0.362 \%$ in adjacent provinces. However, every $1 \%$ increase in AHCR and UR in a province would respectively decrease resident health status $0.125 \%$ and $0.410 \%$ in neighboring provinces. In addition, the spatial lag of RHI was statistically significant and indicated that geographic areas of the spillover effect for resident health status crossed provincial boundaries.

Table 3 Estimates of key human factors direct and indirect effects

\begin{tabular}{|c|c|c|c|c|}
\hline Variable & Direct effect & $P$ value & Indirect effect & $P$ value \\
\hline $\operatorname{lnGDPPC}$ & $-0.018^{* *}$ & 0.038 & $-0.164^{* * *}$ & 0.009 \\
\hline $\ln \mathrm{PCPHE}$ & $-0.016^{* *}$ & 0.045 & 0.009 & 0.747 \\
\hline $\ln \mathrm{AHCR}$ & -0.057 & 0.180 & $0.125^{* *}$ & 0.015 \\
\hline $\operatorname{lnUR}$ & $-0.132^{* *}$ & 0.021 & $0.410^{* * *}$ & 0.001 \\
\hline $\ln E Q I$ & $-0.008^{* *}$ & 0.011 & $-0.362^{* * *}$ & 0.000 \\
\hline
\end{tabular}

Note: ${ }^{* * *} p<0.01 ;{ }^{* *} p<0.05 ;{ }^{*} p<0.1$

\section{Conclusions and outlook}

In this study, regional differences in and key influencing factors on Chinese resident health status were analyzed using the coefficient of variation, Theil index, Exploratory Spatial Data Analysis (ESDA), and Spatial Durbin Model (SDM). The main conclusions are as follows.

(1) From 2003 to 2013, based on the RHI, Chinese resident health status grew at a rate of $26.98 \%$ and the growth rate was higher in the western region than in the eastern and central regions, reaching $32.26 \%$. During the period, regional differences in resident health status generally increased: the coefficient of variation increased $24.15 \%$ and the Theil index increased $72.25 \%$. In general, differences in resident health status between regions tended to decrease, but within regions it tended to expand, especially in the western region.

(2) The eastern region was dominated by above-medium and high health status provinces; the central region was dominated by provinces transforming from above-medium to above-medium and medium health status; and the western region was dominated by provinces transforming from low and below-medium to medium and above-medium health status. The spatial distribution of resident health status generally formed a "T-shaped" pattern and 
presented an "East-Centre-West" stepwise declining pattern and a symmetric declining toward the north and south from the center.

(3) From 2003 to 2013, both the health status hot-spot and cold-spot decreased, while the distribution of resident health status was generally constant, indicating spatial locking or path dependence. The western region was dominated by a stable cold-spot region while the eastern coast was dominated by a stable hot-spot region.

(4) Economic development, public health expenditure, urbanization, environmental quality, and other human factors had a significant impact on resident health status. Among them, increasing economic development level, greater public health expenditures and urbanization, and improved environmental quality resulted in better resident health status. Due to spatial dependency, provincial resident health status created a feedback effect, wherein resident health status in a certain province can influence resident health in neighboring provinces.

As a core problem closely related to human development, health inequality has aroused increasing concern worldwide. Identifying influencing factors on health inequities and resolving them have become popular topics in the discipline of health geography research (Linsheng et al., 2012; Dummer, 2008). With recent research progress showing human impacts on global environmental change, investigating the effects of global environmental change on health should be an objective of the international community (Martens, 1996). Additionally, the International Human Dimension Programme on Global Environmental Change organized the Population Health Advisory Working Group and convened a symposium, "Towards Sustainable Global Health" to systematically focus on the impact of human dimensions on population health (UNU-EHS, 2008). Given this context, this study analyzed the changing trajectories of regional differences in Chinese resident health status from 2003 to 2013 and the impact of key human factors on resident health status using a Spatial Regression Model. The findings should provide a scientific basis for central and local authorities to form appropriate and region-specific medical and health policies. However, due to limited data availability, this study only selected perinatal mortality, maternal mortality, and incidence of infectious diseases to characterize resident health status. Although the findings are in agreement with the conclusions in the relevant literature (Xie, 2011; Li and Xu, 2015; Yang et al., 2017), resident health status is multi-dimensional and additional indicators of resident health status need to be explored in the future. Furthermore, because the sample data and time nodes are relatively small, this study may not fully reveal the spatio-temporal characteristics of Chinese resident health status. Multi-time domain and multi-scale analyses of resident health status should be comprehensively conducted. In addition, this study only addressed the influence of economic development, public health expenditure, accessibility of health care resources, urbanization, and environmental quality on resident health status. In the future, more human factors should be taken into consideration, such as lifestyle or medical systems. Research on the spatialization of human dimensions, such as spatial differences and configuration, and multiple impact mechanisms on resident health status should also be strengthened. Moreover, we should analyze the relationship between resident health status and spatial changes in human dimensions and its coupling mechanism. 


\section{References}

Acheson D, 1998. Independent Inquiry into Inequalities in Health. London: The Stationery Office.

Baguma D, 2017. Public health safety and environment in inadequate hospital and healthcare settings: A review. Public Health, 144: 23-31.

Bai X, Nath I, Capon A et al., 2012. Health and wellbeing in the changing urban environment: Complex challenges, scientific responses, and the way forward. Current Opinion in Environmental Sustainability, 4(4): 465-472.

Boccolini C S, de Souza Jr P R, 2016. Inequities in healthcare utilization: Results of the Brazilian national health survey, 2013. International Journal for Equity in Health, 15(1): 150-157.

Braveman P, 2006. Health disparities and health equity: Concepts and measurement. Annual Review of Public Health, 27(1): 167-194.

Brinda E M, Attermann J, Gerdtham U G et al., 2016. Socio-economic inequalities in health and health service use among older adults in India: Results from the WHO study on global ageing and adult health survey. Public Health, 141: 32-41.

Chen M, Lu D, Zhang H, 2009. Comprehensive evaluation and the driving factors of China's urbanization. Acta Geographica Sinica, 64(4): 387-398. (in Chinese)

Cheng M, Yang M, 2015. Impact of urbanization on the health of Chinese residents: An empirical study based on the provincial panel data. China Population, Resource and Environment, 25(7): 89-96. (in Chinese)

Commission on Social Determinants of Health (WHO CSDH), 2008. Closing the gap in a generation: Health equity through action on the social determinants of health. http://whqlibdoc.who.int/publications/2008/ 9789241563703_eng.pdf.

Du B, Wang X, 2013. Research of evolution, regional difference and influence factor of the inequalities in health of the old. Population Research, 37(5): 81-90. (in Chinese)

Dummer T J, 2008. Health geography: Supporting public health policy and planning. Canadian Medical Association Journal, 178(9): 1177-1180.

Fang P, Dong S, Xiao J et al., 2010. Regional inequality in health and its determinants: Evidence from China. Health Policy, 94(1): 14-25.

Garcia-Subirats I, Vargas I, Mogollón-Pérez A S et al., 2014. Inequities in access to health care in different health systems: A study in municipalities of central Colombia and north-eastern Brazil. Social Science \& Medicine, 13(1): 204-213.

Han Z, Li B, Zhang K, 2015. Evaluation and spatial analysis of the equalization of basic public service in urban and rural areas in China. Geographical Research, 34(11): 2035-2048. (in Chinese)

Hong E, Ahn B C, 2011. Income-related health inequalities across regions in Korea. International Journal for Equity in Health, 10(1): 1-11.

Kirby R S, 2005. Geography of health inequalities in the developed world: Views from Britain and North America. Professional Geographer, 57(4): 615-617.

Lee L F, Yu J, 2010. Some recent developments in spatial panel data models. Regional Science and Urban Economics, 40(5): 255-271.

Lesage J, Pace R K, 2009. Introduction to Spatial Econometrics. New York: CRC Press.

Li H, Yu W, 2013. Effect of the government's health expenditure on the health of rural residents in China. Social Sciences in China, (10): 41-60. (in Chinese)

Li G, Han M, 2015. The impacts analysis of haze pollution on the resident's expenditures of health care. Chinese Health Economics, 34(7): 74-76. (in Chinese)

Li L, Xu R, 2015. The regional difference of the residents' health level in China. Health Economic Research, (1): 14-20. (in Chinese)

Mackenbach J P, Stirbu I, Roskam A J et al., 2008. Socioeconomic inequalities in health in 22 European countries. New England Journal of Medicine, 358(23): 2468-2481.

Martens W J M, 1996. Global atmospheric changes and human health: An integrated modeling approach. Climate 
Research, 6(2): 107-112.

Miao J, Wu X, 2016. Urbanization, socioeconomic status and health disparity in China. Health \& Place, 42: 87-95.

Ming Y, 2007. Research of the trend of infant mortality rate and regional difference in China. Population Research, 33(5): 77-87. (in Chinese)

Mullachery P, Silver D, Macinko J, 2016. Changes in health care inequity in Brazil between 2008 and 2013. International Journal for Equity in Health, 15(1): 140.

National Health and Family Planning Commission (NHFPC), 2015. China Statistical Yearbook of Health and Family Planning (2015). Beijing: Chinese Union Medical University Press. (in Chinese)

Narayan P K, Narayan S, 2008. Does environmental quality influence health expenditures? Empirical evidence from a panel of selected OECD countries. Ecological Economics, 65(2): 367-374.

Pearce J, Dorling D, Wheeler B, 2006. Geographical inequalities in health in New Zealand, 1980-2001: The gap widens. Australian and New Zealand Journal of Public Health, 30(5): 461-466.

Qi L, 2008. Interrelationship between growth, environment and population health: An empirical analysis based on China's provincial data. China Population, Resource and Environment, 18(6): 169-173. (in Chinese)

Smyth F, 2008. Medical geography: Understanding health inequalities. Progress in Human Geography, 32(1): 119-127.

Sundmacher L, Scheller-Kreinsen D, Busse R, 2011. The wider determinants of inequalities in health: A decomposition analysis. International Journal for Equity in Health, 10(1): 2187-2198.

UNU-EHS (United Nations University, Institute for Environment and Human Health), IHDP, 2008. Towards sustainable global health. Source-Publication Series of UNU-EHS, 11.

Van de Poel E, O' Donnel O, Van Doorslaer E, 2012. Is there a health penalty of China's rapid urbanization? Health Economics, 21(4): 367-385.

Wang J, Xu C, 2017. Geodetector: Principle and prospective. Acta Geographica Sinica, 71(1): 116-134. (in Chinese)

Xie E, 2011. Analysis of regional differences in health in China. Journal of Shanxi Finance and Economics University, 33(8): 11-24. (in Chinese)

Xie E, Tu G, 2011. Health achievement index in China: Equity-efficiency tradeoff. China Soft Science, (7): 9-16.

$\mathrm{Xu}$ D, Chen Y, 2010. Analysis on the effect of environmental quality on health expenditures. China Population, Resource and Environment, 20(4): 159-164. (in Chinese)

Yang L, Li H, Li Y et al., 2010. Progress of medical geography and environmental health studies. Progress in Geography, 29(1): 31-44. (in Chinese)

Yang Z, Ao R, Wang N et al., 2017. Spatial-temporal difference characteristic of pollution's health stress of China. Scientia Geographica Sinica, 37(3): 339-346. (in Chinese)

Zatonski W, 2007. The east-west health gap in Europe: What are the causes? European Journal of Public Health, 17(2): 121-121.

Zheng W, Jiang H, Ai H et al., 2015. Analysis of regional inequalities of basic medical resources supply in China. Geographical Research, 34(11): 2049-2060. (in Chinese)

Zhou Z L, Fang Y, Zhou Z Y et al., 2017. Assessing income-related health inequality and horizontal inequity in China. Social Indicators Research, 132: 1-16. 